\title{
The application of artificial intelligence in lung cancer: a narrative review
}

\author{
Huixian Zhang ${ }^{1,2,3}$, Die Meng ${ }^{1,2 \#}$, Siqi Cai ${ }^{1,2 \#}$, Haoyue Guo ${ }^{1,2}$, Peixin Chen ${ }^{1,2}$, Zixuan Zheng ${ }^{1,2}$, Jun Zhu ${ }^{1,2}$, \\ Wencheng Zhao ${ }^{1}$, Hao Wang ${ }^{1,2}$, Sha Zhao ${ }^{1}$, Jia Yu ${ }^{1}$, Yayi He ${ }^{1}$ \\ ${ }^{1}$ Department of Medical Oncology, Shanghai Pulmonary Hospital, Tongji University Medical School Cancer Institute, Tongji University School of \\ Medicine, Shanghai, China; ${ }^{2}$ Tongji University, Shanghai, China; ${ }^{3}$ Department of Medical Oncology, The First Affiliated Hospital of Zhengzhou \\ University, Zhengzhou, China \\ Contributions: (I) Conception and design: Y He, H Zhang, D Meng, S Cai; (II) Administrative support: None; (III) Provision of study materials \\ or patients: Y He, H Zhang, W Zhao; (IV) Collection and assembly of data: All authors; (V) Data analysis and interpretation: All authors; (VI) \\ Manuscript writing: All authors; (VII) Final approval of manuscript: All authors. \\ \#These authors contributed equally to this work. \\ Correspondence to: Yayi He. Department of Medical Oncology, Shanghai Pulmonary Hospital, Tongji University Medical School Cancer Institute, \\ Tongji University School of Medicine, No. 507 Zhengmin Road, Shanghai 200433, China. Email: 2250601@qq.com.
}

Objective: This review was conducted to systematically summarize the progress made by artificial intelligence technology in early screening based medical imaging, pathological diagnosis, genomics inspection, prognostic evaluation, and individual treatment of lung cancer.

Background: Lung cancer has a high mortality rate in China, which is closely related to the fact that most lung cancer patients are not diagnosed until the malignancy is advanced. Challenges remain in the early detection, accurate diagnosis, monitoring and individual treatment of lung cancers. Artificial intelligence has developed to process large amounts of data, from clinical presentations to physiological images, which is essential for solving the complex issues with clinical medicine. Increasing evidence has suggested that artificial intelligence technology provides novel, promising strategies for the diagnosis and treatment of lung cancer.

Methods: A review of literature was conducted in PubMed, EMBASE and Cochrane to identify the latest research on artificial intelligence and lung cancer, and ultimately to generate a narrative review.

Conclusions: Artificial intelligence plays an important role in the imaging inspection, histopathology examination and genomics inspection of lung cancer. In addition, artificial intelligence has the ability to detect a small number of biomarkers, which is conducive to lung cancer monitoring. Moreover, the intelligent treatment of lung cancer has gradually become the trend of future development, whether in internal medicine or surgical treatment. It is believed that artificial intelligence could improve the early diagnosis of lung cancer and assist doctors in treating lung cancer patients individually.

Keywords: Lung cancer; artificial intelligence; early diagnosis; individual treatment

Submitted Dec 07, 2020. Accepted for publication Mar 24, 2021.

doi: $10.21037 /$ tcr-20-3398

View this article at: http://dx.doi.org/10.21037/tcr-20-3398

\section{Introduction}

The leading cause of cancer-related deaths across the world is lung cancer (1-4). Most lung cancer patients are subject to late diagnosis and the lack of efficient treatment (5). More attention needs to be paid to rapid, sensitive and accurate detection techniques. At present, doctors mainly rely on the clinical features of patients and imaging characters to diagnose lung cancer. However, these methods are ineffective in finding lesions timely 


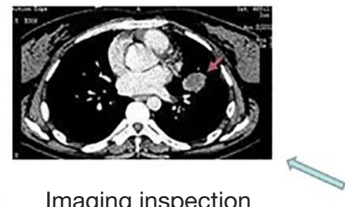

Imaging inspection
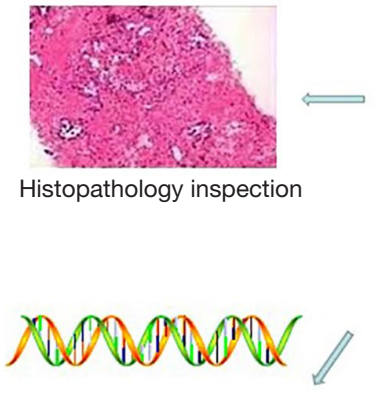

Genomics inspection
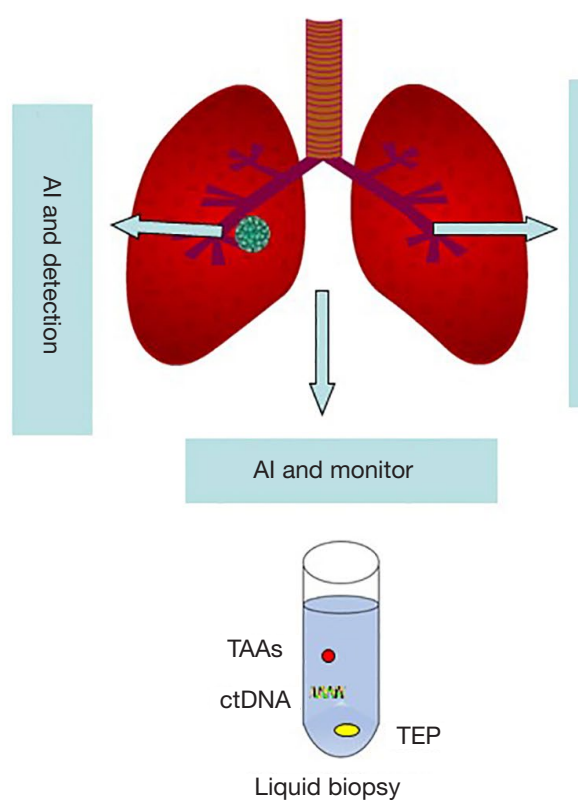

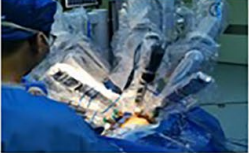

Surgery
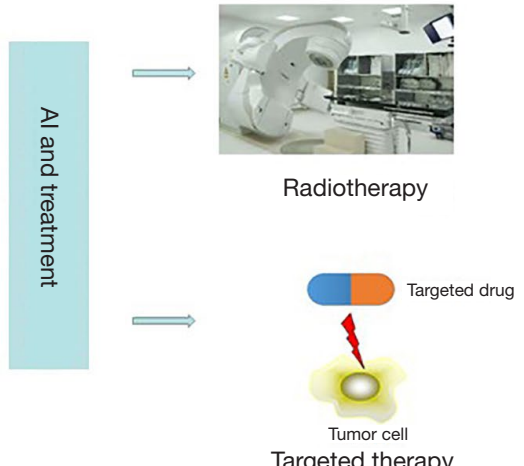

Targeted therapy

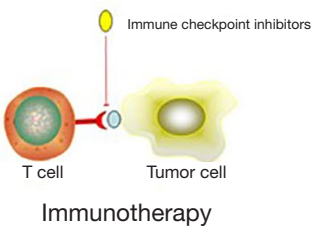

Figure 1 The application of AI in lung cancer: AI plays an important role in the detection, dynamical monitor, and treatment of lung cancer. AI, artificial intelligence; TAAs, tumor-associated antigens; ctDNA, circulating tumor DNA; TEP, tumor educated platelets.

and completely. Artificial intelligence (AI) is referred to as the ability of a digital computer or a robot controlled by a digital computer to carry out some assignments that meet people's needs (6). A large number of studies have demonstrated that $\mathrm{AI}$ is not only valid in detection, dynamical monitor, but also in quantitative treatment during the process of health protection, thus revealing a major capacity for cancer care $(7,8)$. In this review, we will discuss the application of $\mathrm{AI}$ in lung cancer and the challenges in clinical application (Figure 1). We present the following article in accordance with the Narrative Review reporting checklist (available at http://dx.doi.org/10.21037/ tcr-20-3398).

\section{Al and detection}

\section{Imaging inspection}

Clinically, when patients feel uncomfortable or have suspected lesions, their whole body or a certain part will be scanned by means of digital radiography (DR), computed tomography (CT), positron emission computed tomography (PETCT), or magnetic resonance imaging (MRI) $(9,10)$. These imaging techniques as mentioned above play an important role in cancer diagnosis. The main practical problem facing doctors is that their access to information and the integration of digital images of visible anomalies cannot be consistent and accurate $(9,11,12)$.

In an attempt to obtain information instantly with no omission, AI developed its logical algorithm to detect pictures fast. Computer-aided detection (CAD) systems are applied to determine the target site in clinical pictures (13). AI-based detection tools can mark the lesion sites, reduce observational oversights, and make it convenient for readers. For example, CAD systems are easy to distinguish between bronchioles, lung wall and parenchyma clearly while highlighting the lesions that are different than normal, which enables the physicians to ascertain changes without error (12). On the other hand, computer-aided diagnosis systems have paid attention to classifying benign and malignant nodules (14). Up to now, 
new approaches have been designed to detect and diagnose the lung nodules sized less than $3 \mathrm{~mm}$ (15). AI aims to tailor specific solutions to a certain person based on the texture of tumor, character, staging and invasion area.

The imaging features of early lung cancer are pure ground-glass nodule (GGN), partially solid or solid nodule (16). The early detection and surgical resection of lung cancer, presented as GGN or partial solid nodules, can increase the 5 -year survival rate to over $95 \%$ (17). Cancerous pulmonary nodules are difficult to distinguish from benign ones in the early stages. The most suitable solution to lung cancer screening is low-dose CT (LDCT) (18). However, for radiologists, the early detection of lung cancer nodules is very challenging.

The application of AI can overcome the shortage of radiologists and is conducive to the early diagnosis of lung cancer. In recent years, there has been remarkable progress made by the CAD system of pulmonary nodules using traditional machine learning (ML) approaches (19). Recent studies have shown that AI technology in pulmonary nodule imaging is superior to radiologists in detecting the GGN of more than $5 \mathrm{~mm}$ and screening the nodules of $0 \sim 3 \mathrm{~mm}$ (20). However, CAD systems cannot be used alone in clinical practice. Vasopressors (VS)-CAD system, also known as the ClearRead CT software, which was approved by the US Food and Drug Administration (FDA) in 2016, can improve the detectability of small pulmonary nodules by inhibiting the normal structures in the lung, mainly blood vessels (21).

With the development of AI, deep learning (DL) and convolutional neural networks (CNNs) have shown their massive potential in the detection of pulmonary nodules and are effective in segmenting and classifying pulmonary nodules (22). Hua et al. established a DLbased CT detection system for the pulmonary nodules with a sensitivity of $73 \%$ and specificity of $80 \%$, which is superior to the traditional CAD system (23). Ardila and his colleagues developed a DL-based model to predict the risk of developing the lung cancer by analyzing the recent and previous CT volume of patients (24). Nevertheless, detecting pulmonary nodules by AI still needs to be verified in practice and it requires more cases for rectification (25).

\section{Histopathology inspection}

Traditionally, pathological examination has been identified as the most important criterion for the diagnosis of lung cancer, which is an effective approach to digging out changes compared with the surrounding tissue and to identifying the nature of the biopsy species (26-28). However, most patients who undergo biopsies are at an advanced stage and cannot be cured. In addition, what has been taken is just a part of tumor that can't indicate lesions entirely (29). The vast majority of pathological methods are limited to discriminating equivocal histological features, especially in poorly differentiated tumors (9). Drawing upon multiple strands of research into AI, histological analysis can be conducted to develop exhaustive and intelligent diagnostic tactics for biopsy sample (29).

The studies on identifying sensitive and specific method to make diagnosis is widely established. A novel source of large data for personalized medicine was collected by using high-throughput whole-slide scanning technologies and through image computing. Compared with routine clinical images, AI gets integrated with data information prognostically and predicatively to make accurate histology slides analysis (30). Moreover, pathologists can speed up workflow by using the automatic machines that may offer large volume slides and conduct analysis excellently $(9,31)$. Immunohistochemical (IHC) staining that works in mucin staining is an easily available technique that helps to standardize the assessment of the subtype of lung cancer $(29,32)$. In the study of Koh et al., a reliable IHC panel was applied to diagnose the histological subtype of lung cancer thoroughly. They employed two AI approaches including a support vector machine (SVM) and a decision tree to ascertain ambiguous morphology from 30 small biopsy cases of lung cancer. In the absence of 3-marker IHC panel (i.e., TTF-1, Napsin A and p40), they achieved the highest accuracy of approximately $72 \%$ based on the decision tree model that performed two markers (p63 and CK5/6) (33). Although AI expedites workflow and provides precious images to help diagnose lung cancer, the application of AI in clinical settings is rare. The most important reason is that the technology algorithm has not been improved sufficiently to meet the needs of extended patients. In addition, the high price is also a barrier to the widespread application of AI.

\section{Genomics inspection}

With the release of the whole genome sequence and functional genomics data, individualized medical genetics assists with therapy decisions and helps practitioners to prescribe an appropriate therapy for a certain patient. The overexpression of a proto-oncogene or disruption caused by a tumor suppressor gene may lead to cell transformation and tumor formation (34-36). In recent researches, proto- 
oncogenes and tumor-suppressor genes have been identified as markers for the diagnosis of cancer (30). For example, the mutations in the epidermal growth factor receptor (EGFR) gene or rearrangement of the anaplastic lymphoma kinase (ALK) receptor gene are conspicuous in lung cancer, and they are the molecular targets for custom therapeutic selection (37).

The gene conveys semblance of lung cancer by establishing microarrays and AI understands genomic pathways by optimizing algorithm $(38,39)$. Cancer subtype, tumor growth and metastatic potential can be predicted using AI classifiers $(40,41)$. With the growth of the next generation and other methods to reveal the sequences of gene appearance, clinicians will be able to rely on the genomic data to make individual treatment decisions (42). Watson for Genomics (WfG) is being developed to analyze the results of genetic sequencing for patients (43). A study was conducted to evaluate the effectiveness of WfG, in which the gene sequencing results of patients, including lung cancer patients with lung cancer, were first analyzed by hospital multidisciplinary experts and then reanalyzed by WfG (44). According to the results reported, for the 249 oncogenic alterations identified using both approaches, $84.6 \%$ of the targeted therapies proposed by experts were covered by WfG which also provided an additional 225 treatment options.

\section{Al and monitor}

Liquid biopsy, which is distinct from the traditional approaches retrieving biopsy arduously and huge invasively, is developed as a tool for screening. Liquid biopsy makes great contribution to determining the possible genomics alterations which will guide treatment options (6). In recent years, some studies have been conducted to reveal a variety of biomarkers, such as tumor-associated antigens (TAAs), circulating tumor DNA (ctDNA), tumor-associated autoantibodies (TAAbs), tumor educated platelets (TEP), and others (45).

Currently, the studies on liquid biopsy in cancer diagnosis are focused on the AI developed ML to detect a small number of biomarkers. ML is a part of $\mathrm{AI}$ and it is a great algorithm to find millions of microcirculatory genomics fragments that are more sensitive and earlier to be found compared with traditional methods (46). However, due to the limitation of tumor interstitial heterogeneity, there is still a long way to go for the application of liquid biopsy in clinical practice $(11,47)$.

\section{Al and treatment}

With the development of AI, the intelligent diagnosis and treatment of lung cancer has gradually become the trend of future development. Clinical decision support system (CDSS) is a tool to assist clinicians in making clinical decisions through the comprehensive analysis of clinical knowledge (including clinical guidelines, evidence-based medicine, drug instructions, etc.) as well as the basic and clinical information on patients through $\mathrm{AI}$ (48). Watson for Oncology (WFO), as the best representative of CDSS, has been used in the diagnosis and treatment of some cancers, including lung cancer. A retrospective study was conducted to evaluate the consistency of WFO and multidisciplinary teams (MDT) in the treatment of lung cancer cases (49). The results suggested that the overall coincidence rate between MDT and WFO was $92.4 \%$ in lung cancer patients. The treatment recommendations for stage I and IV lung cancers are highly consistent. However, it is relatively low in stage II-III NSCLC and localized small cell lung cancer. In particular, the compliance rate for stage III NSCLC was only $80.8 \%$.

Nevertheless, CDSS cannot completely replace doctors. Instead, it can play a supplementary role, especially for the patients with complex conditions. Moreover, due to the complexity of clinical medicine, doctor-patient communication and shared decision making may play a more important role in treatment.

\section{Surgery}

Video-assisted thoracic surgery (VATS) has been widely applied to treat lung cancer $(50,51)$. A large number of previous studies have demonstrated that compared with thoracotomy, thoracoscopic lobectomy is characterized by less trauma, faster postoperative recovery and fewer complications (52). However, due to the limitations of thoracoscopy and surgical instruments, there will be some blind spots in the operation. In addition, the instrument arm is not as flexible as required to carry out more complex thoracoscopic surgery (53).

AI promises to revolutionize the way surgery is taught and practiced (54). Surgeons play a key role in integrating $\mathrm{AI}$ into clinical practice. The application of $\mathrm{AI}$ is beneficial for surgeons to analyze intraoperative progress in real time, improve the ability of intraoperative decision-making ability and finally improve the surgical outcomes (55). Robot surgery, which belongs to the physical branch of AI, 
is a new direction of development for minimally invasive surgery (56). As a representative of robot surgery, the Da Vinci-robot-assisted thoracic surgery (RATS) has a precise system and convenient operation function, thus breaking the limits of hands and eyes while reducing pain and trauma for patients. The safety and feasibility of the Da Vinci surgical system in lobectomy have been demonstrated in several studies (57-59). A meta-analysis reveals that RATS and VATS are equally effective and safe in treating nonsmall-cell lung cancer, but RATS lasts longer. In addition, there were no statistically significant differences observed between RATS and VATS in terms of intraoperative conversion to thoracotomy rate, the incidence of postoperative complications, postoperative in-hospital mortality, intraoperative blood loss, postoperative drainage days and postoperative hospital stay (58). On the other hand, despite the above-mentioned advantages of RATS, there are some controversies surrounding this technology, such as the higher hospital costs and longer operation time, which may limit the development of RATS.

\section{Radiotherapy}

Chemoradiotherapy is the standard treatment for nonresectable advanced non-small cell lung cancer (NSCLC) $(60,61)$. The combination of the AI and radiation contributes much in the development of radiology, which is conducive to realizing precision treatment $(62,63)$. In a study, a clinical model of stereotactic body radiotherapy (SBRT) was designed for lung cancer patients using knowledge-based algorithms to plan treatment (64). In this study, 105 SBRT plans to treat lung cancer patients were involved, including 97 intensity modulated radiotherapy (IMRT), 6 volumetric arc radiotherapy (VMAT), and 2 three-dimensional conformal radiationtherapy (CRT). Knowledge-based model (KBM) training was carried out by combining the forementioned technologies, and multiple verification results showed that SBRT plans for lung cancer generated by KBM was comparable to clinical plans.

Existing radiation techniques are designed to eliminate tumors, but they often cause damage to healthy tissue. The application of AI will solve the problem effectively and be able to adjust the volume of treatment. In addition, the optimal angle and radiation beam can be selected automatically without being influenced by the posture of patients (65). Furthermore, intelligent system has the ability to regulate irradiation time, dose rate and images. It is believed that physicians will make plans rapidly and preciously using AI $(66,67)$.

\section{Targeted therapy}

Targeted therapy is a new approach to treat cancer with minimal damage to normal tissues by targeting molecules on tumor cells and developing effective blockers to intervene in the carcinogenesis process (68-70). Determining which location changed and what happened in the mutation region is an important step when we want to proceed. AI provides a large amount of data and enables an in-depth investigation, which supports us in finding various ways to prepare for useful option. In addition, intelligent machine establishes related model to find targeted gene or relational molecular pathway and make it visible.

With the assistance of AI, newly discovered therapeutic targets can be virtually screened in a very short time to find targeted old drugs or new compounds (71). AI-assisted reverse docking could be highly effective in reapplying old drugs or natural products to new indications (72). The characteristics of absorption, distribution, metabolism, excretion and toxicity (ADMET) can also be predicted by the DL model of AI, so as to improve the success rate of in vivo experiments (73).

\section{Immunotherapy}

The evaluation of immunotherapy for immune-responsive malignancies, particularly lung cancer, shows significant promise (74). Immune checkpoint inhibitors (ICIs), such as inhibitors of programmed death-1 (PD-1), cytotoxic correlative antigen-4 (CTLA-4), and programmed deathligand 1 (PD-L1), exert prospective effects in advanced lung cancer (75-77).

However, some challenging problems arising from this domain are patient selection and immune response $(78,79)$. Most of prior researches focused on predictive biomarkers about histological and genetic markers from biopsies (80). Charoentong et al. made a major contribution to setting up a score scheme using AI that can be applied to predict the response of patients to ICIs (81). In addition, Coroller et al. used CT data to demonstrate the radiomic feature after treatment (82).

The Keynote-042 study revealed that the expression of PD-L1 was closely related to the curative effect, and that the higher the expression level of PD-L1, the more significant the benefit of immunotherapy in NSCLC patients (83). The detection method of PD-L1 is simple, 
convenient, straightforward, and less demanding on dyeing technology. However, there are still many problems, for example, the imbalance of detection method, strong subjectivity in the doctor's interpretation and the difficulty in giving quantitative data. AI technology has been successfully applied to the segmentation and recognition of tumor cells in tumor pathological images. Besides, it can also accurately quantify the results of IHC staining. Therefore, AI can detect the expression of PD-L1 in lung cancer tissues objectively and repeatably, which is conducive to predicting the efficacy of immunotherapy (84).

More effective biomarkers than PD-L1 are needed to predict efficacy. The toughest challenge for immunotherapy is that the process of treatment response is difficult to monitor because of dynamic changes. Once someone accepts immunotherapy, the following variation can hardly be ascertained accurately and completely. Although immunotherapy is hard to meet the final goals currently, precision treatment is made promising by the rapid development of AI (85).

\section{Future challenges}

The application of $\mathrm{AI}$ in understanding and treating lung cancer is advancing, but there still remain many obstacles to overcome. In healthcare settings, AI should follow unified planning and total target, as well as normalized and standard principle established by the authority that provides refined details to practice in real situation easily (62).

$\mathrm{ML}$ is a way to implement $\mathrm{AI}$, which learns from examples rather than being programmed. Generally speaking, more examples are required for ML than human to master the same skill (56). Therefore, data acquisition is also of great importance. It is an easy way to obtain the medical records information of patients from electronic health records, which demonstrate all detailed information of the patient in hospital. Because of different recording requirements in disparate departments or districts, however, a large number of fragmented records present a challenge to the clinical application of AI (86). Moreover, language and culture barriers, patient privacy, sample volumes are all obstacles to obtaining available data $(87,88)$.

\section{Conclusions}

The aspiration to improve the efficacy and efficiency of health care continues to drive diverse creations into practice, including AI. It has developed widespread and is essential for solving complex issues in multiple areas. Herein, we explained multiple applications of $\mathrm{AI}$ in the care of lung cancer patients. Currently, the researches on $\mathrm{AI}$ in oncology mainly focus on diagnosis, dynamic monitoring and personal treatment. By using intelligent machines to process vast amounts of data, from clinical presentations to physiological images, physicians can make decisions quickly and accurately. Moreover, computer-assisted diagnosis will help to detect early lesions, which is beneficial to the prognosis of patients. Due to the lack of standard databases, standardization principles formulated by authoritative institutions, and corresponding national policies and regulations, the application of $\mathrm{AI}$ in lung cancer has a broad prospect, despite a long way to go. To realize the goal of individual treatment, more sensitive and precise machines or algorithms are needed.

\section{Acknowledgments}

Funding: This study was supported in part by a grant of National Natural Science Foundation of China (81802255), Clinical research project of Shanghai Pulmonary Hospital (FKLY20010), Young Talents in Shanghai (2019 QNBJ), 'Dream Tutor' Outstanding Young Talents Program (fkyq1901), Clinical research project of Shanghai Pulmonary Hospital (FKLY20001), Respiratory medicine, a key clinical specialty construction project in Shanghai, Promotion and application of multidisciplinary collaboration system for pulmonary non infectious diseases, Clinical Research Project of Shanghai Pulmonary Hospital (fk18005), Key Discipline in 2019 (oncology), Project of Shanghai Municipal Science and Technology Commission (Project of Municipal Science and Technology Commission), Scientific research project of Shanghai Pulmonary Hospital (fkcx1903), Shanghai Municipal Commission of Health and Family Planning (2017YQ050), Innovation Training Project of SITP of Tongji University, and key projects of leading talent (19411950300). Youth project of hospital management research fund of Shanghai Hospital Association (Q1902037).

\section{Footnote}

Reporting Checklist: The authors have completed the Narrative Review reporting checklist. Available at http:// dx.doi.org/10.21037/tcr-20-3398

Conflicts of Interest: All authors have completed the ICMJE 
uniform disclosure form (available at http://dx.doi. org/10.21037/tcr-20-3398). The authors have no conflicts of interest to declare.

Ethical Statement: The authors are accountable for all aspects of the work in ensuring that the questions related to the accuracy or integrity of any part of the work are appropriately investigated and resolved.

Open Access Statement: This is an Open Access article distributed in accordance with the Creative Commons Attribution-NonCommercial-NoDerivs 4.0 International License (CC BY-NC-ND 4.0), which permits the noncommercial replication and distribution of the article with the strict proviso that no changes or edits are made and the original work is properly cited (including links to both the formal publication through the relevant DOI and the license). See: https://creativecommons.org/licenses/by-nc-nd/4.0/.

\section{References}

1. Sonego M, Pellizzari I, Dall'Acqua A, et al. Common biological phenotypes characterize the acquisition of platinum-resistance in epithelial ovarian cancer cells. Sci Rep 2017;7:7104.

2. Krasnov GS, Puzanov GA, Afanasyeva MA, et al. Tumor suppressor properties of the small C-terminal domain phosphatases in non-small cell lung cancer. Biosci Rep 2019;39:BSR20193094.

3. Ferlay J, Soerjomataram I, Dikshit R, et al. Cancer incidence and mortality worldwide: sources, methods and major patterns in GLOBOCAN 2012. Int J Cancer 2015;136:E359-86.

4. Roth C, Kasimir-Bauer S, Pantel K, et al. Screening for circulating nucleic acids and caspase activity in the peripheral blood as potential diagnostic tools in lung cancer. Mol Oncol 2011;5:281-91.

5. Guo H, Chen X, Su C, et al. Challenges and countermeasures of thoracic oncology in the epidemic of COVID-19. Transl Lung Cancer Res 2020;9:337-47.

6. O'Keeffe M, Barratt A, Maher C, et al. Media Coverage of the Benefits and Harms of Testing the Healthy: a protocol for a descriptive study. BMJ Open 2019;9:e029532.

7. Viscaino M, Maass JC, Delano PH, et al. Computer-aided diagnosis of external and middle ear conditions: A machine learning approach. PLoS One 2020;15:e0229226.

8. Li SM, Chen CH, Chen YW, et al. Upregulation of CISD2 augments ROS homeostasis and contributes to tumorigenesis and poor prognosis of lung adenocarcinoma. Sci Rep 2017;7:11893.

9. Rabbani M, Kanevsky J, Kafi K, et al. Role of artificial intelligence in the care of patients with nonsmall cell lung cancer. Eur J Clin Invest 2018;48.

10. Shi Z, Song T, Wan Y, et al. A systematic review and metaanalysis of traditional insect Chinese medicines combined chemotherapy for non-surgical hepatocellular carcinoma therapy. Sci Rep 2017;7:4355.

11. Bi WL, Hosny A, Schabath MB, et al. Artificial intelligence in cancer imaging: Clinical challenges and applications. CA Cancer J Clin 2019;69:127-57.

12. Fraioli F, Serra G, Passariello R. CAD (computedaided detection) and $\mathrm{CADx}$ (computer aided diagnosis) systems in identifying and characterising lung nodules on chest CT: overview of research, developments and new prospects. Radiol Med 2010;115:385-402.

13. Brinkløv S, Kalko EK, Surlykke A. Intense echolocation calls from two 'whispering' bats, Artibeus jamaicensis and Macrophyllum macrophyllum (Phyllostomidae). J Exp Biol 2009;212:11-20.

14. Keshani M, Azimifar Z, Tajeripour F, et al. Lung nodule segmentation and recognition using SVM classifier and active contour modeling: a complete intelligent system. Comput Biol Med 2013;43:287-300.

15. Gong J, Liu J, Hao W, et al. Computer-aided diagnosis of ground-glass opacity pulmonary nodules using radiomic features analysis. Phys Med Biol 2019;64:135015.

16. Matsuguma H, Mori K, Nakahara R, et al. Characteristics of subsolid pulmonary nodules showing growth during follow-up with CT scanning. Chest 2013;143:436-43.

17. Henschke CI, Yip R, Smith JP, et al. CT Screening for Lung Cancer: Part-Solid Nodules in Baseline and Annual Repeat Rounds. AJR Am J Roentgenol 2016;207:1176-84.

18. Aberle DR, Adams AM, Berg CD, et al. Reduced lungcancer mortality with low-dose computed tomographic screening. N Engl J Med 2011;365:395-409.

19. Goo JM. Computer-aided detection of lung nodules on chest CT: issues to be solved before clinical use. Korean J Radiol 2005;6:62-3.

20. Lo SB, Freedman MT, Gillis LB, et al. JOURNAL CLUB: Computer-Aided Detection of Lung Nodules on CT With a Computerized Pulmonary Vessel Suppressed Function. AJR Am J Roentgenol 2018;210:480-8.

21. Milanese G, Eberhard M, Martini K, et al. Vessel suppressed chest Computed Tomography for semiautomated volumetric measurements of solid pulmonary nodules. Eur J Radiol 2018;101:97-102. 
22. Tandon YK, Bartholmai BJ, Koo CW. Putting artificial intelligence (AI) on the spot: machine learning evaluation of pulmonary nodules. J Thorac Dis 2020;12:6954-65.

23. Hua KL, Hsu CH, Hidayati SC, et al. Computer-aided classification of lung nodules on computed tomography images via deep learning technique. Onco Targets Ther 2015;8:2015-22.

24. Ardila D, Kiraly AP, Bharadwaj S, et al. End-to-end lung cancer screening with three-dimensional deep learning on low-dose chest computed tomography. Nat Med 2019;25:954-61.

25. Wan YL, Wu PW, Huang PC, et al. The Use of Artificial Intelligence in the Differentiation of Malignant and Benign Lung Nodules on Computed Tomograms Proven by Surgical Pathology. Cancers (Basel) 2020;12:2211.

26. Yang D, Li R, Zhang XH, et al. Perfusion Characteristics of Hepatocellular Carcinoma at Contrast-enhanced Ultrasound: Influence of the Cellular differentiation, the Tumor Size and the Underlying Hepatic Condition. Sci Rep 2018;8:4713.

27. Yuan Z, Quan J, Yunxiao Z, et al. Diagnostic value of contrast-enhanced ultrasound parametric imaging in breast tumors. J Breast Cancer 2013;16:208-13.

28. Liang H, Fu M, Zhou J, et al. Evaluation of 3D-CPA, HR-HPV, and TCT joint detection on cervical disease screening. Oncol Lett 2016;12:887-92.

29. Shulimzon TR. Endomicroscopy, Not "Optical Biopsy" (Yet). Am J Respir Crit Care Med 2017;195:962.

30. Djuric U, Zadeh G, Aldape K, et al. Precision histology: how deep learning is poised to revitalize histomorphology for personalized cancer care. NPJ Precis Oncol 2017;1:22.

31. Shillan D, Sterne JAC, Champneys A, et al. Use of machine learning to analyse routinely collected intensive care unit data: a systematic review. Crit Care 2019;23:284.

32. Jia K, He Y, Dziadziuszko R, et al. T cell immunoglobulin and mucin-domain containing-3 in non-small cell lung cancer. Transl Lung Cancer Res 2019;8:895-906.

33. Koh J, Go H, Kim MY, et al. A comprehensive immunohistochemistry algorithm for the histological subtyping of small biopsies obtained from non-small cell lung cancers. Histopathology 2014;65:868-78.

34. Sun Y, Zhu S, Ma K, et al. Identification of 12 cancer types through genome deep learning. Sci Rep 2019;9:17256.

35. Wang Z, Sun Y. Targeting p53 for Novel Anticancer Therapy. Transl Oncol 2010;3:1-12.

36. Tanaka M, Kato K, Gomi K, et al. NU'T midline carcinoma: report of 2 cases suggestive of pulmonary origin. Am J Surg Pathol 2012;36:381-8.
37. Lawrence MS, Stojanov P, Polak P, et al. Mutational heterogeneity in cancer and the search for new cancerassociated genes. Nature 2013;499:214-8.

38. Ohashi K, Sequist LV, Arcila ME, et al. Lung cancers with acquired resistance to EGFR inhibitors occasionally harbor BRAF gene mutations but lack mutations in KRAS, NRAS, or MEK1. Proc Natl Acad Sci U S A 2012;109:E2127-33.

39. Koivunen JP, Mermel C, Zejnullahu K, et al. EML4-ALK fusion gene and efficacy of an ALK kinase inhibitor in lung cancer. Clin Cancer Res 2008;14:4275-83.

40. Kikuchi T, Daigo Y, Katagiri T, et al. Expression profiles of non-small cell lung cancers on cDNA microarrays: identification of genes for prediction of lymph-node metastasis and sensitivity to anti-cancer drugs. Oncogene 2003;22:2192-205.

41. Haack H, Johnson LA, Fry CJ, et al. Diagnosis of NUT midline carcinoma using a NUT-specific monoclonal antibody. Am J Surg Pathol 2009;33:984-91.

42. Podolsky MD, Barchuk AA, Kuznetcov VI, et al. Evaluation of Machine Learning Algorithm Utilization for Lung Cancer Classification Based on Gene Expression Levels. Asian Pac J Cancer Prev 2016;17:835-8.

43. Chen Y, Yan W, Xie Z, et al. Comparative analysis of target gene exon sequencing by cognitive technology using a next generation sequencing platform in patients with lung cancer. Mol Clin Oncol 2021;14:36.

44. Itahashi K, Kondo S, Kubo T, et al. Evaluating Clinical Genome Sequence Analysis by Watson for Genomics. Front Med (Lausanne) 2018;5:305.

45. Revelo AE, Martin A, Velasquez R, et al. Liquid biopsy for lung cancers: an update on recent developments. Ann Transl Med 2019;7:349.

46. Chew RF, Amer S, Jones K, et al. Residential scene classification for gridded population sampling in developing countries using deep convolutional neural networks on satellite imagery. Int J Health Geogr 2018;17:12.

47. Lim ZF, Ma PC. Emerging insights of tumor heterogeneity and drug resistance mechanisms in lung cancer targeted therapy. J Hematol Oncol 2019;12:134.

48. Klarenbeek SE, Weekenstroo HHA, Sedelaar JPM, et al. The Effect of Higher Level Computerized Clinical Decision Support Systems on Oncology Care: A Systematic Review. Cancers 2020;12:1032.

49. Kim MS, Park HY, Kho BG, et al. Artificial intelligence and lung cancer treatment decision: agreement with recommendation of multidisciplinary tumor board. Transl 
Lung Cancer Res 2020;9:507-14.

50. Raman V, Christopher JK, Jawitz OK, et al. Robot- vs Video-Assisted Thoracoscopic Lobectomy for Early Lung Cancer. JNCI Cancer Spectr 2020;4:pkaa031.

51. Wu L, Wang H, Cai H, et al. Comparison of Double Sleeve Lobectomy by Uniportal Video-Assisted Thoracic Surgery (VATS) and Thoracotomy for NSCLC Treatment. Cancer Manag Res 2019;11:10167-74.

52. Paul S, Altorki NK, Sheng S, et al. Thoracoscopic lobectomy is associated with lower morbidity than open lobectomy: a propensity-matched analysis from the STS database. J Thorac Cardiovasc Surg 2010;139:366-78.

53. Arad T, Levi-Faber D, Nir RR, et al. The learning curve of video-assisted thoracoscopic surgery (VATS) for lung lobectomy--a single Israeli center experience. Harefuah 2012;151:261-5, 320.

54. Hashimoto DA, Rosman G, Rus D, et al. Artificial Intelligence in Surgery: Promises and Perils. Ann Surg 2018;268:70-6.

55. Mangano A, Valle V, Dreifuss NH, et al. Role of Artificial Intelligence (AI) in Surgery: Introduction, General Principles, and Potential Applications. Surg Technol Int 2020;38:sti38/1369.

56. Rajkomar A, Dean J, Kohane I. Machine Learning in Medicine. N Engl J Med 2019;380:1347-58.

57. Veronesi G, Galetta D, Maisonneuve P, et al. Four-arm robotic lobectomy for the treatment of early-stage lung cancer. J Thorac Cardiovasc Surg 2010;140:19-25.

58. Guo F, Ma D, Li S. Compare the prognosis of Da Vinci robot-assisted thoracic surgery (RATS) with video-assisted thoracic surgery (VATS) for non-small cell lung cancer: A Meta-analysis. Medicine (Baltimore) 2019;98:e17089.

59. Liu X, Xu S, Liu B, et al. Survival Analysis of Stage I Nonsmall Cell Lung Cancer Patients Treated with Da Vinci Robot-assisted Thoracic Surgery. Zhongguo Fei Ai Za Zhi 2018;21:849-56.

60. Wang CH, Lin CY, Chen JS, et al. Karnofsky Performance Status as A Predictive Factor for Cancer-Related Fatigue Treatment with Astragalus Polysaccharides (PG2) Injection-A Double Blind, Multi-Center, Randomized Phase IV Study. Cancers (Basel) 2019;11:128.

61. Nakashima T, Huang C, Liu D, et al. Neural-cadherin expression associated with angiogenesis in non-small-cell lung cancer patients. Br J Cancer 2003;88:1727-33.

62. Lambin P, Leijenaar RTH, Deist TM, et al. Radiomics: the bridge between medical imaging and personalized medicine. Nat Rev Clin Oncol 2017;14:749-62.

63. Aerts HJ. The Potential of Radiomic-Based Phenotyping in Precision Medicine: A Review. JAMA Oncol 2016;2:1636-42.

64. Chin Snyder K, Kim J, Reding A, et al. Development and evaluation of a clinical model for lung cancer patients using stereotactic body radiotherapy (SBRT) within a knowledge-based algorithm for treatment planning. J Appl Clin Med Phys 2016;17:263-75.

65. Amit G, Purdie TG, Levinshtein A, et al. Automatic learning-based beam angle selection for thoracic IMRT. Med Phys 2015;42:1992-2005.

66. Liu Z, Wang S, Dong D, et al. The Applications of Radiomics in Precision Diagnosis and Treatment of Oncology: Opportunities and Challenges. Theranostics 2019;9:1303-22.

67. Hosny A, Parmar C, Quackenbush J, et al. Artificial intelligence in radiology. Nat Rev Cancer 2018;18:500-10.

68. Jackisch C, Ruschoff J, Ullrich A. Development of Targeted Therapies in ErbB2-Positive Breast Cancer. Breast Care (Basel) 2008;3:3-6.

69. Sai C, Wang J, Li B, et al. Isolation and identification of alkaloids from Macleaya microcarpa by UHPLC-Q-TOF$\mathrm{MS}$ and their cytotoxic activity in vitro, antiangiogenic activity in vivo. BMC Chem 2020;14:5.

70. He Y, Zhou C. Tyrosine kinase inhibitors interstitial pneumonitis: diagnosis and management. Transl Lung Cancer Res 2019;8:S318-20.

71. Zhavoronkov A, Ivanenkov YA, Aliper A, et al. Deep learning enables rapid identification of potent DDR1 kinase inhibitors. Nat Biotechnol 2019;37:1038-40.

72. Talele TT, Khedkar SA, Rigby AC. Successful applications of computer aided drug discovery: moving drugs from concept to the clinic. Curr Top Med Chem 2010;10:127-41.

73. Liu B, He H, Luo H, et al. Artificial intelligence and big data facilitated targeted drug discovery. Stroke Vasc Neurol 2019;4:206-13.

74. Teeling EC, Springer MS, Madsen O, et al. A molecular phylogeny for bats illuminates biogeography and the fossil record. Science 2005;307:580-4.

75. Evans AG, French CA, Cameron MJ, et al. Pathologic characteristics of NUT midline carcinoma arising in the mediastinum. Am J Surg Pathol 2012;36:1222-7.

76. Finlay WJJ, Coleman JE, Edwards JS, et al. Anti-PD1 'SHR-1210' aberrantly targets pro-angiogenic receptors and this polyspecificity can be ablated by paratope refinement. MAbs 2019;11:26-44.

77. Prendergast CT, Sanin DE, Cook PC, et al. CD4+ T cell hyporesponsiveness after repeated exposure to Schistosoma 
mansoni larvae is dependent upon interleukin-10. Infect Immun 2015;83:1418-30.

78. Wolchok JD, Hoos A, O'Day S, et al. Guidelines for the evaluation of immune therapy activity in solid tumors: immune-related response criteria. Clin Cancer Res 2009; 15:7412-20.

79. Hodi FS, Hwu WJ, Kefford R, et al. Evaluation of Immune-Related Response Criteria and RECIST v1.1 in Patients With Advanced Melanoma Treated With Pembrolizumab. J Clin Oncol 2016;34:1510-7.

80. He Y, Zhang X, Jia K, et al. OX40 and OX40L protein expression of tumor infiltrating lymphocytes in nonsmall cell lung cancer and its role in clinical outcome and relationships with other immune biomarkers. Transl Lung Cancer Res 2019;8:352-66.

81. Charoentong P, Finotello F, Angelova M, et al. Pancancer Immunogenomic Analyses Reveal GenotypeImmunophenotype Relationships and Predictors of Response to Checkpoint Blockade. Cell Rep 2017;18:248-62.

82. Coroller TP, Agrawal V, Narayan V, et al. Radiomic phenotype features predict pathological response in non- small cell lung cancer. Radiother Oncol 2016;119:480-6.

83. Mok TSK, Wu YL, Kudaba I, et al. Pembrolizumab versus chemotherapy for previously untreated, PD-L1expressing, locally advanced or metastatic non-small-cell lung cancer (KEYNOTE-042): a randomised, open-label, controlled, phase 3 trial. Lancet 2019;393:1819-30.

84. Koelzer VH, Sirinukunwattana K, Rittscher J, et al. Precision immunoprofiling by image analysis and artificial intelligence. Virchows Arch 2019;474:511-22.

85. Mehnert JM, Monjazeb AM, Beerthuijzen JMT, et al. The Challenge for Development of Valuable Immunooncology Biomarkers. Clin Cancer Res 2017;23:4970-9.

86. Schmidt C. M. D. Anderson Breaks With IBM Watson, Raising Questions About Artificial Intelligence in Oncology. J Natl Cancer Inst 2017;109.

87. Leyens L, Reumann M, Malats N, et al. Use of big data for drug development and for public and personal health and care. Genet Epidemiol 2017;41:51-60.

88. Auffray C, Balling R, Barroso I, et al. Making sense of big data in health research: Towards an EU action plan. Genome Med 2016;8:71.
Cite this article as: Zhang H, Meng D, Cai S, Guo H, Chen P, Zheng Z, Zhu J, Zhao W, Wang H, Zhao S, Yu J, He Y. The application of artificial intelligence in lung cancer: a narrative review. Transl Cancer Res 2021;10(5):2478-2487. doi: 10.21037/ tcr-20-3398 